\title{
EVALUATION OF SUBJECTIVE PREFERENCES B Y FUZZY COGNITIVE MAPS OF SEMI-AUTONOMOUS DECISION MAKING SYSTEMS
}

\author{
Martin Gavalec* \\ Karel Mls \\ Faculty of Informatics and Management \\ University of Hradec Králové \\ Hradec Králové, Czech Republic \\ E-mail: martin.gavalec@uhk.cz \\ E-mail:karel.mls@uhk.cz
}

\begin{abstract}
Cognitive maps in semi-autonomous systems subsuming supervisor, decisional agent and its environment are considered. The goals specified by the supervisor influence the subjective preferences of the decisional agent. Multi-criteria AHP method is used to determine the behavior of the agent. Evaluation of the subjective preferences of the agent is modified so that the operational goals will lead to realization of the given strategic goals. Computation of the modif ied subjective preferences is described in the paper.
\end{abstract}

Keywords: AHP, FCM, autonomous system, multi-criteria decision making

\section{Introduction}

Selecting the best option among several possible ones, or adopting a working solution in limited time, are usual problems people often face. By creating more and more complicated and sophisticated systems we are approaching to the point, when the system management by humans becomes practically impossible, and autonomous or semi-autonomous behavior of artificial entities will inevitably join the human society.

The paper is focused on cognitive maps in semi-autonomous systems. Definition of the elementary semiautonomous decision system subsuming supervisor, decisional agent and its environment will be introduced. The goals set by the supervisor have impact on the subjective preferences of the decisional agent. Thus, the actions of the agent depend not only on its internal (operational) goals, but they also reflect the given higher (strategic) goals. Multi-criteria AHP method will be used here to determine the decision making behavior of the agent.

Evaluation of the subjective preferences of the agent must be modified so that the operational goals will lead to realization of the given strategic goals. Computation of the modified subjective preferences described in the paper is based on the internal states in the agent's fuzzy cognitive map, and on the relative importance of the higher goals.

\footnotetext{
* Corresponding author
} 


\section{Notions}

\subsection{Fuzzy cognitive map}

The concept of Fuzzy cognitive map (FCM) was introduced by Kosko (Kosko, 1986) as a generalization of Axelrod's cognitive map (Axelrod, 1976). Fuzzy logic helps to solve problems with respect to nonprecise humans' way of thinking. Simple FCM works on the principle that concepts and the causal relationships are accompanied by a number within the real unit interval $\langle 0,1\rangle$, or symmetric interval $\langle-1,1\rangle$. By this evaluation, fine dependencies in causal relationships can be expressed and partial activation of concepts can be used, in contrary to the binary activation in CM. Theoretical basis of FCMs has been subsequently elaborated by many authors, (Kim \& Lee, 1998) and (Park \& Kim, 1995) for example.

Alternatively, FCM may be considered as a neuro-fuzzy system (Salmeron, 2009). It is possible to represent the different relationships among concepts by means of a matrix $\mathbf{A}$, called the adjacency matrix:

$$
\mathbf{A}=\left[\begin{array}{ccc}
\ldots & \ldots & \ldots \\
\ldots & e_{i j} & \ldots \\
\ldots & \ldots & \ldots
\end{array}\right] ; \quad e_{i j} \in\langle-1,+1\rangle \quad \forall i, j,
$$

where $e_{i j}$ indicates the relationship between the $i$ and $j$ concepts, enabling us to obtain values between $\langle-1,1\rangle$. Three types of relationships can be seen: $\left.1 . e_{i j}\right\rangle$, indicating a positive relationship, $2 . e_{i j}<0$, indicating a negative one, and 3. $e_{i j}=0$, where no relationship exists. Three basic issues must be considered, when an expert assigns a value $e_{i j}$. Firstly, the $e_{i j}$ intens ity to indicate how strong the $i$ concept is in $j$. Secondly, the sign (+/-) of $e_{i j}$ must be decided to indicate if the relationship between the $i$ and $j$ concepts is direct or inverse. Lastly, the causality relationship needs to be indicated to establish if the $i$ concept is a cause of $j$ or vice-versa. Various methodologies could be used in order to reach a consensus among the experts (if the experts are the data source). For several non-human sources, the Augmented FCM approach must be adopted, as an iterative process has not sense. The augmented adjacency matrix is built adding the adjacency matrix of each source.

Let assume two FCMs with no common nodes: $F C M^{A}$ with $\left\{c_{i}^{A}\right\}$ as nodes, and $F C M^{B}$ with $\left\{c_{j}^{B}\right\}$ as nodes. Note that each FCM can have a different node set. The adjacency matrix of $F C M^{A}$ is $A^{A}=\left(w_{i j}^{A}\right)$; and the adjacency matrix of $F C M^{B}$ is $A^{B}=\left(w_{k m}^{B}\right)$. The augmented adjacency matrix is

$$
A=\left[\begin{array}{cc}
e_{i j}^{A} & 0 \\
0 & e_{k m}^{B}
\end{array}\right]
$$

If there are common nodes, then the element $e_{i j}^{A u g}$ in the augmented matrix is

$$
e_{i j}^{A u g}=\frac{\sum_{k=1}^{n} e_{i j}^{k}}{n},
$$


where $n$ stands for the number of FCMs added, one by data source, $k$ the identifier of each source, and $i$ and $j$ the identifier of the relationships.

In this paper, a FCM is formally defined as an ordered pair $\mathbf{M}=(\boldsymbol{C}, \boldsymbol{A})$, where $\boldsymbol{C}$ is a finite set of cardinality $|\boldsymbol{C}|=n$, whose elements are called concepts, and $\boldsymbol{A}$ is a matrix of type $n \times n$ with values in the real interval $\langle 0,1\rangle$ (alternatively, in $\langle-1,1\rangle$ ).

Elements of matrix $\boldsymbol{A}$ are interpreted as the levels of causal relations between pairs of concepts in $\boldsymbol{C}$. Further, we shall consider an evaluation vector of the fuzzy cognitive map $\boldsymbol{M}$, defined as a mapping $e$ : $\boldsymbol{C}$ $\rightarrow\langle 0,1\rangle$ and its values are interpreted as activation levels of concepts in $\boldsymbol{C}$.

Decision support, and model behavior prediction as well, represent the most often cited doma ins of FCMs utilization, see (Khan, Chong \& Gedeon, 2000), (Salmeron, 2009). FCMs as a supporting tool for decision making process were considered in (Gavalec, \& Mls, 2003).

\subsection{Analytic Hierarchy Process}

Analytic Hierarchy Process (AHP) is a method developed for creating structured models of multi-criteria decision problems. The method helps to find an alternative which suits best the given needs of the deciding person. Analyzing the set of possible alternatives, the AHP method finds the one with the best rating, based on the structure of the problem and given preferences. Saaty formulated the principles of AHP in late 1970s (Saaty, 1980), and the method has been broadly studied and applied in many cases since the time. The method combines mathematical and psychological aspects, starting with defining the structure of the problem, then quantifying the relative preferences, computing the priorities and finally computing the evaluation of all considered alternatives.

First of all, the multi-criteria decision problem is converted into a hierarchy of sub-problems and each of the sub-problems is then independently analyzed. The criteria of the sub-problems in the hierarchy may have very heterogeneous nature, they may be precisely or vaguely defined, with crisp or fuzzy parameters, formal or intuitive, etc. The relative preferences of heterogeneous criteria are then quant if ied by human decision-maker using the ability to compare various aspects of the problem. The decision maker systematically compares given criteria in pairs and quantifies the relative importance either by available data or by intuitive judgment. The relative preferences found by pairwise comparisons are then used to compute weights (priorities) for every part of the hierarchy model.

The evaluation computed for all decision alternatives then shows their relative strength from the point of view of the entire problem. It is the advantage of AHP that even considerably diverse criteria can be used in the model, and that not only exact data but also human judgments can be applied to describe various aspects of the problem.

Formally, AHP is expressed by matrices and matrix operations are used to find and evaluate the best alternative:

Let $A_{1}, A_{2}, . ., A_{\mathrm{n}}$ be a set of variables. The quantified judgments on pairs of variables $A_{i}, A_{j}$ are represented by an $n$-by- $n$ matrix $\mathbf{A}=\left(a_{i j}\right), i, j=1,2, \ldots, n$. Entries $a_{\mathrm{ij}}$ are defined as follows: If $a_{i j}=a$, then $a_{j i}=1 / a ; a$ $\neq 0$ for all $i, j$. As $A_{i}$ is considered to be of equal relative intensity to itself, then $a_{i i}=1$ for all $i$.

Then, relative rankings of variables will be obtained by computing an eigenvector $\mathbf{X}$ of the matrix $\mathbf{A}$.

$$
\mathbf{A X}=\lambda \mathbf{X}
$$


Finally, by matrix multiplication of matrices of relative rankings of criteria and alternatives relative rankings matrix, total ranking of particular alternatives is obtained.

\subsection{Semi-autonomous system}

By an elementary semi-autonomous decision system we understand a triple consisting of a decisional agent, the environment and the agent's supervisor.

An agent is an encapsulated computational (or physical, even human) system, that is situated in some environment, and that is capable of flexible, autonomous behavior in order to meet its design objective (Wooldridge, 2000). The agent can exist on its own but often is a component of a multi-agent system.

There are the following key properties of an autonomous intelligent agent: autonomy, reactivity, intentionality and social capability. Behavior of embodied agents can be described by an agent program, a mapping of agent's percepts to actions (Russel, Norvig, 2003).

By agent autonomy we understand, that the agent is accountable for execution of its own actions and is not controlled from outside. Often the agent's reasoning mechanism that selects the action to be executed is unknown from outside of the agent (unlike e.g. objects). In reality, strictly autonomous behavior is not usual. Agents communicate and use shared data for optimizing of the ir actions. When the communication is not symmetrical and agents are not at the same level in the hierarchy, partial-autonomous or semiautonomous behavior of the agent can be observed. Semi-autonomous agent is then accountable for execution of its actions, but can be partially controlled by other agent or agents.

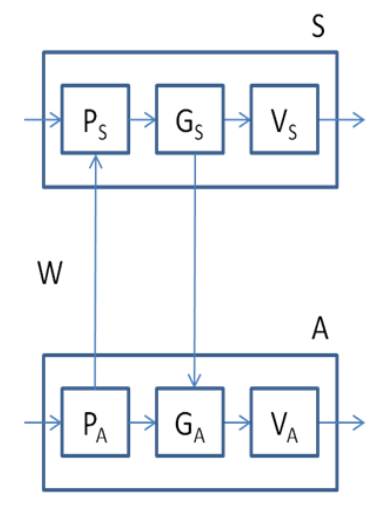

Figure 1. Agent and supervisor in semi-autonomous system (P - perceptions, G - goals, V - actions, W - environment)

\section{Subjective preferences of the agent}

The agent behavior is determined by its internal model. The behavior is influenced and triggered by the current state of the environment, represented by the set of parameters, which can be sensed by appropriate sensors and mediated to the agent as perceptions.

Agent's own decisional preferences are considered to be encoded in the structure of the FCM model, particularly these preferences may be identified from the state vector of the FCM as the response to the perceptions from the environment.

The FCM weights may be subject of change in time, too, as the model is allowed to evolve with respect to level of understanding of the environment (e.g. due to obtaining new information from the environment or due to changes of environment itself). 


\section{Behavior of the agent}

By behavior we understand continuous selecting appropriate action from the set of all possible actions. Priorities play important role in agent's decision making if it is based on multi-criteria method. In the semi-autonomous system, priorities of the agent and the supervisor are proposed to merge in the FCM model and direct the resulting agent's behavior. Agent is processing information from the environment and looking for optimal action from its local perspective (local goals), and in the same time it is receiving supervisor's demands (global goals), influencing the resulting decision of the agent. Resulting behavior is then a balanced compromise between agent's and supervisor's goals, or operational realization of tactical or strategic goals.

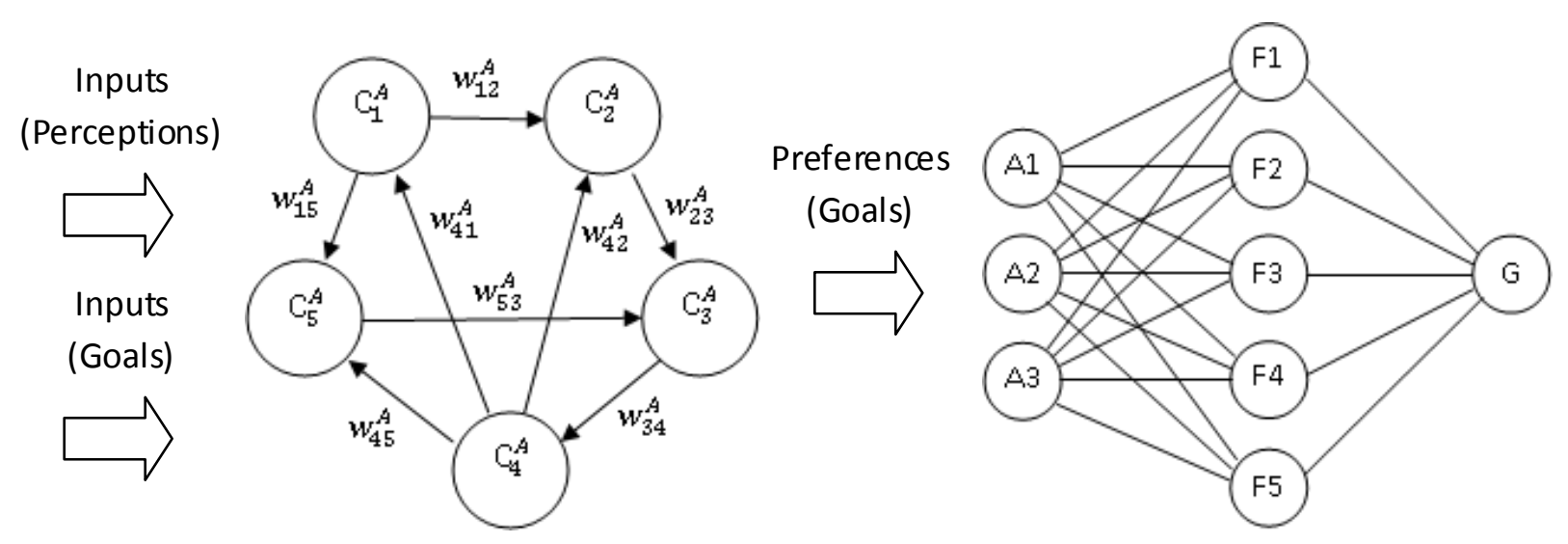

Figure 2. Agent's behavior model and its decisional hierarchy (A - Alternatives/actions, F - Factors/criteria, G - Goa1/action)

\section{Conclusions}

Fuzzy cognitive map together with Analytic hierarchy process were suggested as a method for decision making of the semi-autonomous agent. While agent's perception of the environment is transformed by the FCM to agent's preferences and leads to finding the best action from the operational perspective, and supervisor's goals leads to the best action from the strategic perspective, by merging the agent's and supervisor's FCMs it is possible to reach globally optimal behavior of the agent.

Further research will be necessary for deeper understanding of learning processes in the agent-supervisorenvironment system, and for automatic optimization of the ratio between agent-supervisor preferences.

The support of Czech Science Foundation GAČR projects \#402/09/0405 and \#402/09/0662 is kindly acknowledged.

\section{REFERENCES}

Axelrod, R. (1976). Structure of Decision: The Cognitive Maps of Political Elites. Princeton University Press, New Jersey.

Gavalec, M., \& Mls, K. (2003). Fuzzy cognitive maps and decision making support. Proc. of the 21st Conf. Mathem. Methods in Economics, Czech University of Agriculture in Prague, 87-93. 
Khan, M.S., Chong, A., \& Gedeon, T. (2000). A Methodology for Developing Adaptive Fuzzy Cognitive Maps for Decision Support. Journal of Advanced Computational Intelligence, 4(6), 403-407

Kim, H., \& Lee, K. (1998). Fuzzy implications of fuzzy cognitive map with emphasis on fuzzy causal relationship and fuzzy partially causal relationship. Fuzzy Sets and Systems. 97(3), 303-313.

Kosko, B. (1986). Fuzzy cognitive maps. International Journal of Man-Machine Studies, 24(1), 65-75.

Park, K., \& Kim, S. (1995). Fuzzy Cognitive Maps Considering Time Relationships. International Journal of Human-computer Studies, 42(2), 157-168.

Russel, S., \& Norvig, P. (2003). Artificial Intelligence A Modern Approach, 2nd edition. Prentice Hall.

Saaty, T.L. (1980). The analytic hierarchy process. McGraw-Hill, New York.

Salmeron, J.L. (2009). Supporting decision makers with Fuzzy Cognitive Maps. Research-Technology Management, 52(3), 53-59.

Wooldridge, M. (2000). Reasoning about rational agents. MIT Press, London. 